\title{
EVALUACIÓN DE PROTOCOLOS DE SEGUIMIENTO DE VÍAS VENOSAS PERIFÉRICAS. NEONATOLOGÍA. HOSPITAL DR. HERNÁN HENRÍQUEZ ARAVENA. MAYO- JUNIO DE 2005.
}

\author{
EVALUATION OF PROTOCOLS OF FOLLOWING OF OPERIPHERICAL \\ CATHETER VENOUS. NEONATOLOGY. DR. HERNAN HENRIQUEZ ARAVENA \\ HOSPITAL. MAY- JUNE OF 2005.
}

ANGÉLICA RIVAS LIENQUEO* EDITH RIVAS RIVEROS**

\begin{abstract}
RESUMEN
Objetivo: conocer las causas que se asocian a multipunción en el recién nacido, con el propósito de focalizar estrategias de mejoramiento. Material y Método: Diseño de corte transversal y asociación. Muestra: 371 protocolos clínicos de vías venosas periféricas; variables: edad gestacional, motivo de retiro, horas de duración. Resultados: $60,65 \%$ de las vvp corresponden a recién nacidos mayores de 1500 grs. y $39,35 \%$ a recién nacidos de muy bajo peso de nacimiento $(<1500 \mathrm{grs})$. El $64,42 \%$ de las vías, se instala en primera punción y $35,58 \%$ requiere más de un intento. En viabilidad de las vías venosas periféricas, $68,45 \%$ permanece in situ más de 24 horas, 30,45\% más de 48 horas, 1,08\% menos de una hora y 22,37\% entre 49 y 72 horas. En recién nacidos pretérmino, el 62,68\% de las vías venosas periféricas duró menos de 72 horas in situ y en el recién nacido de término, 37,32\%, p: 0.038. Motivo de retiro de las vías: $34,77 \%$ por extravasación, $15,36 \%$ por término de uso, $10,51 \%$ por vencimiento y flebitis 12,67\%. Conclusiones: Existe un elevado porcentaje de multipunción, extravasación y limitada duración. Se debe considerar movilidad del neonato, inmovilización, características de la piel y la calidad de los insumos.
\end{abstract}

Palabras claves: Multipunción, instalación vía venosa periférica, neonato.

\begin{abstract}
Objective: to know the causes associated to multi venipucture site in the NB, in order to focus improvement strategies. Material and Method: Design of cross section and association. Sample: 371 clinical protocols of pvc; variables: gestacional age, remove reason, hours of duration. Results: $60,65 \%$ of pvc correspond to more than 1500 grs NB. And $39,35 \%$ to VLBWI (<1500grs). $64,42 \%$ of the catheter, is settle in a first puncture and $35,58 \%$ require more than one attempt. In viability of pvc, $68,45 \%$ stay in situ more than 24 hours, $30,45 \%$ more than 48 hours, $1,08 \%$ less than one hour and 22,37\% between 49 and 72 hours. In PI the $62,68 \%$ of pvc lasted less than 72 hours in situ and in the TI 37,32\%, p: 0.038 . Reason of the replacement central: $34,77 \%$ by extrusion, $15,36 \%$ by cease, $10,51 \%$ by expiration and phlebitis $12,67 \%$. Conclusions: high percentage of multi venipucture, extrusion and limited duration. To consider mobility of the newborn, immobilization, characteristics of the skin and supplies quality.
\end{abstract}

Keywords: Multi venipucture, peripheral central venous, pcv installation, newborn.

Fecha Recepción: 19 diciembre 2007 Fecha Aceptación: 29 septiembre 2008

*EU. Licenciada en Enfermería, Unidad de Cuidados Intensivos, Neonatología, Hospital Dr. Hernán Henriquez Aravena. Temuco. Chile. E-mail: ayrivasl@gmail.com

**EU, Magíster en Salud Pública, Departamento de Pediatría y Cirugía Infantil, Facultad de Medicina, Universidad de La Frontera. Temuco. Chile.E-mail: erivas@ufro.cl 


\section{INTRODUCCIÓN}

El acceso venoso periférico es la intervención de mayor uso, para la instalación de terapia intravenosa en el cuidado de neonatos en unidades de cuidado intensivo neonatal (UCIN). Es una intervención determinante, debido a que su instalación puede disminuir el riesgo de muerte de un recién nacido $(\mathrm{RN})$ y es un procedimiento ineludible y rutinariamente empleado en neonatos de cuidado intensivo, dada la necesidad de aportar líquidos precozmente, nutrición parenteral o fármacos en general, como antibióticos y fármacos vasoactivos (Ducks, 1997 In: Barría \& Santander, 2006). Lo anterior exige efectuar una adecuada técnica de instalación que permita un acceso seguro, duradero y que no genere complicaciones al neonato.

La obtención de un acceso venoso periférico, es un procedimiento invasivo y muchas veces traumático para el neonato (De Lima, Lloyd-Thomas, Howard, Summer \& Quinn, 1996), es una indicación médica específica, para la toma de exámenes, terapias antibióticas o no antibióticas, reanimación, monitorización y debe ser suspendido cuando el motivo de instalación ha cesado (Comité de Infecciones Intrahospitalarias, Temuco-Chile, 2005). El procedimiento, no está exento de complicaciones y no siempre se obtiene de primera intención, lo que puede conducir a multipunción; situación que debe llamar a la reflexión, pues se trata del bienestar de los pacientes, de disminuir el riesgo el dolor y de reconocer nuestras limitaciones, para mejorar la calidad de atención. La evaluación del dolor, en los neonatos es un desafío para el profesional de enfermería (Puchalski \& Hummel, 2002), el que debe manejar habilidades intelectuales, relacionales, afectivas y de comunicación, de modo de aliviar y otorgarle el más completo bienestar al Recién Nacido (Clifford, Stringer, Chiristensen, \& Mountain, 2004; Goubert et al., 2005).

En el manejo del dolor en neonatos, se han producido cambios significativos y han aumentado notablemente el número de publicaciones científicas. Cabrero, Orts, LópezCoig, Velasco y Richard (2005) se refieren a que ya no se debate si el recién nacido siente dolor o no. Por muchos años, se manejaron conceptos erróneos acerca de la percepción y experiencia dolorosa del RN, tal como que su sistema nervioso central era inmaduro y no contaba con las estructuras necesarias para la percepción del estímulo doloroso (Pasero, 2004; Puchalski \& Hummel, 2002). Sin embargo, hoy se sabe que el neonato percibe el dolor y que esta sensación puede influir en conductas regresivas o demandantes que alteran su relación con el entorno a edades futuras (Martinez-Tellería, Delgado, Cano , Núñez \& Gálvez, 2002; Young, 2005)

Recientemente, un grupo internacional de expertos ha elaborado un documento de consenso basado en revisiones sistemáticas con recomendaciones detalladas en relación con procedimientos dolorosos específicos y guías que pueden ser de utilidad a los profesionales sanitarios que debemos asistir a recién nacidos (Consensus Statement for the prevention and management of pain in the newborn, 2001). Las medidas generales (American Academy of Pediatrics. Committee on Fetus and Newborn, 2000), se centran en prevenir, sobre todo evitando el estímulo doloroso recurrente y minimizando los procedimientos dolorosos (valorar el procedimiento menos doloroso, sustituir medidas invasivas por no invasivas) y, cuando se pueda, coordinar con otros aspectos del cuidado del niño; disminuir estímulos agresivos innecesarios.

De esta forma, se plantea el objetivo de conocer las causas que se asocian a multipunción en el RN, con el propósito de focalizar estrategias de mejoramiento y de esta manera acentuar la calidad del procedimiento. Es de especial relevancia, aún más si se tiene en cuenta que los Neonatos son totalmente dependientes de nuestro accionar, ética y responsabilidad profesional. En este sentido, la Reforma del sector Salud es insoslayable: equidad en el acceso, eficiencia, calidad en las 
prestaciones y protección de la dignidad de las personas. Estos procesos políticos y sociales, requieren de decisiones técnicas certeras y fundadas en evidencia científica indiscutible. Es responsabilidad del mundo académico hacer su aporte, generando esas evidencias a través de una investigación científica rigurosa y de calidad, que de cuenta de su pertinencia social (Enríquez, 2004).

Otras complicaciones reportadas se refieren a hematomas, flebitis, infección, sepsis y eventos tromboembólicos (Hermansen \& Hermansen, 2005). Conjuntamente la infección nosocomial ha sido fuertemente vinculada a algunos procedimientos invasivos y uso de dispositivos como los catéteres venosos periféricos (Beck-Sague et al., 1994; Stoll et al., 2002; Urrea et al., 2003). Al respecto Mac Queen (2005), ha planteado la relación entre estas infecciones y la edad, (mayor incidencia en neonatos y lactantes menores).

De acuerdo a lo anterior, surge la necesidad de investigar, a través de los protocolos de seguimiento las causas que se asocien a multipunción en el RN, con el propósito de orientar directrices en el juicio profesional y en la gestión del cuidado, contribuyendo a mejorar la calidad de atención de la Enfermería Neonatal y la seguridad del paciente, colaborando en las nuevas políticas de salud.

\section{MATERIAL Y MÉTODO}

Diseño de corte transversal y de asociación, realizado en la Unidad de Neonatología Dr. Hernán Henríquez Aravena de la ciudad de Temuco, IX Región de Chile, durante el período Mayo- Junio de 2005. Su objetivo fue determinar causas de multipunción de los neonatos, con el fin de mejorar la calidad de atención de Enfermería.

La muestra estuvo constituida por 371 protocolos, diseñados y validados por expertos. El análisis se realizó con el programa STATA 8.0 (Stata Corporation, College Station, Texas, USA 2003) y se utilizaron, en variables cuali- tativas: distribuciones de frecuencias relativas y absolutas y en variables cuantitativas: media y desviación estándar. Para establecer asociaciones de variables se utilizó la prueba $\chi^{2}$ El nivel de significación utilizado en las pruebas estadísticas fue de un 5\%.

\section{RESULTADOS}

Fueron estudiados 371 protocolos, que correspondieron a $97 \mathrm{RN}, 60,65 \%$ registraron un peso de más de 1500 grs. y $61,19 \%$ eran menores de 37 semanas de edad gestacional, superando en un $22,38 \%$ a los de término $(38,81 \%)$.

El 61,19\%, de las vías venosas instaladas, se utilizaron para fleboclisis y $38,81 \%$ para tapón in luer. $91,54 \%$ de los Recién Nacidos se encontraba con indicación de antibioterapia.

23 protocolos $(6,20 \%)$ presentaban indicación de transfusión, para lo cual se instaló acceso vascular periférico exclusivo para esta infusión. $64,42 \%$ de las vías se instaló de primera intención y $35,58 \%$ requirió más de una punción. El 63,88\% de los protocolos, revelaron que los Recién Nacidos fueron puncionados una vez, $26,42 \%$ dos veces y $1,62 \%$ cuatro veces por un mismo profesional para la instalación de una vía venosa.

El 68,45\% de las vías presentó una duración de más de 24 horas, 30,45\% alcanzó a estar in situ más de 48 horas y 1,08\% menos de una hora. El promedio de duración de las vvp fue de 37,95 horas con una DE $\pm 21,98$. $34,77 \%$ de las vías debió ser reinstalada por extravasación, $12,67 \%$ por flebitis y $11,32 \%$ por salida accidental. $15,36 \%$, se retiraron por término de uso y $10,51 \%$ por vencimiento de las vías.

En los neonatos de término, $57,14 \%$ de sus vías presentó una duración de más de 72 horas y $37,32 \%$ menos de 72 horas. Se estableció significancia estadística entre la duración de las vías venosas y la edad gestacional de los neonatos.

Dentro de los factores de riesgo asociados a 
multipunción se consideró el uso de antibióticos, sin embargo al establecer relación entre el uso de estos y la duración de las vías venosas no se encontró un valor significativo.

\section{DISCUSIÓN}

El mantenimiento de la permeabilidad de los catéteres venosos periféricos (CVP), es una práctica clínica común en los pacientes que precisan medicación, administración de fluidos o derivados sanguíneos. Sin embargo, no hay directrices universales sobre el modo más adecuado de realizarla (Deeb \& Di Mattia, 1976). Estudios de la American Association of Critical Care Nurses (AACCN, 1990), de Cabrero (2005), de Batton, Maisels y Appelbaum (1982) y de Fernández, Griffiths y Murie (2003); plantean interrogantes respecto del mantenimiento de la permeabilidad de los CVP en la práctica habitual y concluyen la existencia de una gran variabilidad en la práctica del procedimiento, asociada a la realidad local.

En el número de punciones por RN, $63,88 \%$ fueron puncionados una vez, $26,42 \%$ dos veces y $2,7 \%$ cuatro y más, por un mismo profesional, para la instalación de una vía. La extravasación, flebitis y filtraciones pueden explicar la necesidad de volver a puncionar al Recién Nacido, pero no deja entrever la licencia para multipunción. Dada su condición de procedimiento invasivo y traumático para el neonato, debe ser justificado su uso, por el riesgo asociado. En la práctica clínica se valida que un mismo profesional realice no más de tres intentos de canalización, situación que está sujeta al criterio personal y al cumplimiento del principio ético de no maleficencia. Cuando no se logra canalizar una vía venosa a la tercera punción, el profesional debe solicitar a su par que realice el procedimiento. Al respecto, un estudio de Alvarez y Tiberio (2000), señala que en procedimientos menores, en los cuales la posibilidad de fallar es alta, se aconseja emplear una buena técni- ca, utilizar instrumentos adecuados, aplicar anestésicos locales, colocar lentamente y con cuidado las sondas y, en caso necesario, sobre todo en niños ventilados sometidos a procedimientos de radiografía, aplicar una dosis extra de morfina.

El promedio de duración de las vías en la unidad estudiada fue de 37,95 horas con una $\mathrm{DE} \pm 21,98$, siendo de 11 horas menos que los resultados obtenidos en un estudio randomizado, controlado, (Batton, Maisels \& Appelbaum, 1982) donde las vías venosas periféricas presentaron una duración promedio de 49,5 horas y DE de $\pm 30,9$ horas y las causas de retiro fueron $57 \%$ por filtración y $37 \%$ por reacción inflamatoria no relacionada a infección.

Al respecto Riquelme, Babiano, Palma, Avendaño y Novoa (2000) evaluaron en la unidad de Neonatología del Hospital Padre Hurtado, el manejo de Enfermería de las vías venosas en Puerperio para administración de ATB, y se encontró un promedio de duración de 63 horas con mediana de 72 horas y el 55\% de los Recién Nacidos requirió instalar una segunda vía debido a un $68 \%$ de filtración, $16 \%$ vía tapada, $13 \%$ mala fijación y un caso por no corresponder el retiro.

Para favorecer la viabilidad de las vías venosas y evitar multipuncionar a los neonatos, en algunos centros del país y extranjero se utiliza el flash intermitente con heparina; sin embargo, en un estudio realizado en Canadá se evaluó la administración de infusión continua y flasheo con heparina y no se encontró diferencia en el tiempo de duración (Prakesh et al, 2007)

Referente al motivo de reemplazo de las vías, el mayor porcentaje se lo adjudica la extravasación (34,77\%). Aquí puede considerarse la movilidad del neonato, la técnica de inmovilización, las características de la piel y la calidad de los insumos. Dichas cifras son similares a las encontradas por Garland et al. (1992), donde se examinaron las complicaciones y asociación con factores de riesgo. Encontrándose una recurrencia de flebitis 
del $13 \%$, extravasación $28 \%$ y colonización por bacterias $11 \%$. Cabe hacer mención que en este estudio, se monitorizaron vías periféricas con duración de 48 a 144 horas.

Un estudio de Barría y Santander (2006), encontró como principal motivo de retiro para cánulas de Vialon y agujas de acero tipo mariposa, la extravasación en casi $75 \%$ de los casos.

El retiro electivo, por término de la terapia, cumplimiento de la vía o no uso del catéter se presentó en $13,4 \%$ de los catéteres Insyte y $14,6 \%$ de las agujas tipo mariposa.

De acuerdo a los resultados obtenidos al cruzar las variables relacionadas con factores de riesgo de multipunción: edad gestacional, uso de antibióticos, horas de duración de las vías, profesional instalador, numero de intentos de punción y lugar de instalación no se encontró significancia estadística. Sólo se apreció significancia estadística entre la utilización de ayudante para la instalación - duración de vía venosa y edad gestacional - duración de vía venosa (ambas variables independientes).

En el ámbito de la calidad asistencial, el rol de la Enfermera en la práctica clínica y administrativa adquiere relevancia la problemática de los registros. Nuestro estudio arrojó hallazgos respecto al déficit en registros por parte de los profesionales de Enfermería, (20,1\%), lo cual se evidencia en protocolos con datos incompletos, los que fueron excluidos para el estudio. Situación que dificulta el desarrollo de la enfermería basada en la evidencia (EBE), ya que un número considerable de estudios quedan limitados debido a la falta de información tangible. La EBE permite a la disciplina aumentar su cuerpo de conocimientos a través de la investigación y en la práctica clínica, permite tomar decisiones con base científica, entregando cuidados óptimos a personas que los necesitan (Orellana \& Paravic, 2007). Sin embargo, es difícil encontrar dichas competencias en una sola persona, por lo que las enfermeras con aptitudes y preparación en investigación deben trabajar en conjunto con las enfermeras clínicas, para aumentar las posibilidades de éxito en la implementación de las evidencias (Lacasaña, 2006).

La competencia profesional para el cuidado demanda un ejercicio crítico, reflexivo y constructivo sobre las prácticas disciplinares e interdisciplinares en salud, siendo capaz de conocer e intervenir en el proceso de vivir, enfermar y estar sano, con compromiso y responsabilidad en la búsqueda del mejoramiento de la vida. El trabajo de enfermería se centra en el cuidado, en las dimensiones de cuidar, gerenciar, educar e investigar, sustentado por los diversos patrones de conocimientos, competencias y habilidades específicas (Mendes, Trevizan \& Lourenco, 2002; Trevizan \& Mendes, 1993; Trevizan \& Mendes, 1995).

En las variables estudiadas, obviamos la descripción del género de los Recién Nacidos; si bien estos datos pudieron haberse inferido del nombre del neonato, no todos los protocolos contaban con esta información.

Finalmente con el propósito de focalizar estrategias de mejoramiento se sugiere que los profesionales de enfermería ahonden en la temática de acuerdo a sus realidades locales, y en factores de riesgo modificables tales como: calidad de los insumos, efectividad de la inmovilización de las vías venosas, habilidad y conducta ética del profesional, entre otras.

De igual forma se sugiere el seguimiento de las vías venosas, debido al riesgo de complicaciones que inherentemente conlleva su instalación y que generan repercusión como indicador de calidad. Se requiere realizar auditorias, periódicas de los protocolos, valorando su duración y complicaciones en la instalación.

\section{REFERENCIAS}

Álvarez \& Tiberio. (2000). Dolor en los neonatos. Enfoque diagnóstico y terapéutico. Latreia, 13 (4), 246-256

American Academy of Pediatrics. Committee on Fetus and Newborn. Committee on Drugs. Section on Anesthesiology. Section on Surgery. 
Canadian Pediatric Society. Fetus and Newborn Committee. Prevention and Management of pain and stress in the Neonate. (2000). Pediatrics, 105454-61

American association of critical care nurses. AACN (1990). Nationwide practice survey results announced. New, 3 .

Barría, M \& Santander, G. (2006). Acceso vascular periférico en neonatos de cuidado intensivo: experiencia de un hospital público. Cienc. Enferm, 12, (2), 35-44.

Batton, D.G., Maisels, M.J. \& Appelbaum, P. (1982). Use of peripheral intravenous cannulas in premature infants: a controlled study. Pediatrics, 70, 487-490.

Beck-Sague, C.M.; Azimi, P.; Fonseca, S.N.; Baltimore, R.S.; Powell, D.A.; Bland, L.A. et al. (1994). Bloodstream infections in neonatal intensive care unit patients: results of a multicenter study. Pediatr. Infect, 13, 1, 110-1.116.

Cabrero J, Orts MI, López-Coig ML, Velasco ML, Richard M.(2005) Variabilidad en la práctica clínica del mantenimiento de la permeabilidad de los catéteres venosos periféricos. Gac Sanit, 19, 287-293.

Clifford, P. A., Stringer, M., Christensen, H., \& Mountain, D. (2004). Pain Assessment and intervention for term newborns. J Midwifery Womens Health, 49, (6), 514-519.

Comité de Infecciones Intrahospitalarias. (2005) Normas de Infecciones Intrahospitalarias. Temuco- Chile, 11-13.

Consensus Statement for the prevention and management of pain in the newborn.Anand KJS(2001). Arch Pediatr Adolesc Med, 155, 173-80.

De Lima J, Lloyd-Thomas AR, Howard RF, Summer E \& Quinn TM. (1996) Infant and neonatal pain: Anesthetists, perceptions and prescribing pattern. $B M J, 3,787$.

Deeb E. \& Di Mattia P. (1976). How much heparin in the lock. Am J Intravenous Ther, 3, 22-6.

Enríquez, O. (2004) Enfermería, Investigación y Reforma de Salud. Cienc. Enferm., 10, (1), 7-7.

Fernandez R S, Griffiths R D, Murie P (2003). Peripherical venous catheters: a review of current practices. J Infus Nurs, 26, 388-92.

Garland, J.S.; Dunne, W.M., Jr.; Havens, P.; Hintermeyer, M.; Bozzette, M.A.; Wincek, J. et al. (1992). Peripheral intravenous catheter com- plications in critically ill children: a prospective study. Pediatrics, 89, 1.145-1.150.

Goubert, L., Craig, K. D., Vervoort, T., Morley, S., Sullivan, M. J., de, C.W.A.C., et al. (2005). Facing others in pain: the effects of empathy. Pain, 118(3), 285-288.

Hermansen, M.C. \& Hermansen, M.G. (2005). Intravascular catheter complications in the neonatal intensive care unit. Clin. Perinatol, 32, 141-56.

Lacasaña, P. (2006). ¿Y después de la investigación qué? Reflexión sobre la implementación de la evidencia en la práctica clínica enfermera. Evidentia.3(10). Recuperado el 16 de junio de 2006 de http://www.mdex- f.com/evidentia / n10/2 36artículo. php

Mac Queen, S. (2005). The special needs of children receiving intravenous therapy. Nurs. Times 101, 59, 61- 64.

Martinez-Tellería A, Delgado JA, Cano ME, Núñez J \& Gálvez R (2002).: Postoperative analgesia in the neonate. Revista Sociedad Española Dolor; 9, $317-327$.

Mendes, I. A. C., Trevizan, M. A. \& Lourenço, M. R. (2002). A gerência esclarecida no trabalho do enfermeiro. Revista Latino-americana de Enfermagem 10, (5), 704-708.

Orellana, A. \& Paravic, T. (2007). Enfermería basada en evidencia: barreras y estrategias para su implementación. Cienc. Enferm., 13, (1), 17-24.

Pasero, C. (2004). Pain relief for neonate. Am J Nurs, 104(5), $44-47$.

Prakesh S., et al. (2007) Trial of Heparin Versus Placebo Infusion to Prolong the Usability of Peripherally Placed Percutaneous Central Venous Catheters (PCVCs) in Neonates: The HIP (Heparin Infusion for PCVC).StudyPediatrics, 119, 284 - 291.

Puchalski, M., \& Hummel, P. (2002). The reality of neonatal pain. Adv Neonatal Care, 2(5), 233-244; 245-237.

Riquelme E., Balbiano M.A., Palma D., Avendaño G., Novoa J., (2000). Evaluación de vías venosas en Recién Nacidos de Puerperio. Hospital Padre Hurtado. SSMSO. Unidad de Neonatología, Santiago - Chile en Resúmenes de Trabajos presentados en el Xl Congreso Chileno De Pediatría I Congreso Chileno De Adolescencia. Rev. chil. Pediatr,71, (6), 520-567. 
Stoll, B.J.; Hansen, N.; Fanaroff, A.A.; Wright, L.L.; Carlo, W.A.; Ehrenkranz, R.A. et al. (2002). Late-onset sepsis in very low birth weight neonates: the experience of the NICHD Neonatal Research Network. Pediatrics 110, 285-291.

Trevizan, M. A., Mendes, I. A. C. (1993). Administration of patient care: theoretical aspects. International Nursing Review, 40, (1), 25-28.
Trevizan, M.A., Mendes, I.A.C. (1995). Perspectives of participative management in nursing. Applied Nursing Research, 8, (4), 156-159.

Urrea, M.; Iriondo, M.; Thio, M.; Krauel, X.; Serra, M.; Latorre, C. et al. (2003). A prospective incidence study of nosocomial infections in a neonatal care unit. Am. J. Infect, 31, 505-507.

Young, K. D. (2005). Pediatric procedural pain. Ann Emerg Med, 45(2), 160-171. 